\title{
Correction to: European multicentre study on technical success and long-term clinical outcome of radiofrequency ablation for the treatment of spinal osteoid osteomas and osteoblastomas
}

\author{
Thomas Beyer $^{1}$ - Carla S. P. van Rijswijk ${ }^{2}$ - Jose Martel Villagrán ${ }^{3} \cdot$ Christoph Rehnitz $^{4}$ - Mario Muto ${ }^{5}$. \\ Christian von Falck ${ }^{6} \cdot$ Jan Gielen $^{7} \cdot$ Kolja M. Thierfelder ${ }^{1} \cdot$ Marc-André Weber $^{1,4}$
}

Published online: 19 June 2019

(C) Springer-Verlag GmbH Germany, part of Springer Nature 2019

\section{Correction to: Neuroradiology 2019}

https://doi.org/10.1007/s00234-019-02226-9

The original version on this paper contained an error: the Acknowledgements were dropped during early manuscript editing. The missing statement is presented below:

Acknowledgements This study is endorsed by the European Society of Neuroradiology (ESNR), the European Society of Musculoskeletal Radiology (ESSR), the German Society of Musculoskeletal Radiology (DGMSR) and the German Radiological Society (DRG).

Publisher's note Springer Nature remains neutral with regard to jurisdictional claims in published maps and institutional affiliations.

The online version on the original article can be found at https://doi.org/ 10.1007/s00234-019-02226-9

Thomas Beyer

thomas.beyer@med.uni-rostock.de

1 Institute of Diagnostic and Interventional Radiology, Paediatric Radiology and Neuroradiology, University Medical Centre Rostock, Ernst-Heydemann-Str. 6, 18057 Rostock, Germany

2 Department of Radiology, Leiden University Medical Centre, Leiden, The Netherlands

3 Diagnóstico por Imagen, Hospital Universitario Fundación Alcorcón, Alcorcón, Madrid, Spain

4 Department of Diagnostic and Interventional Radiology, Heidelberg University Hospital, Heidelberg, Germany

5 Neuroradiology Department, Cardarelli Hospital, Naples, Italy

6 Department of Diagnostic and Interventional Radiology, Hannover Medical School, Hannover, Germany

7 Department of Radiology, Antwerp University Hospital, Antwerp, Belgium 